\title{
Non-SARS-CoV-2 genome sequences identified in clinical samples from COVID-19 infected patients: Evidence for co- infections
}

\author{
Mohamed A Abouelkhair ${ }^{\text {Corresp. } 1}$ \\ ${ }^{1}$ Department of Biomedical and Diagnostic Sciences College of Veterinary Medicine, University of Tennessee, Knoxville, Tennessee, United States \\ Corresponding Author: Mohamed A Abouelkhair \\ Email address: mabouelk@vols.utk.edu
}

\section{Background}

In December 2019, an ongoing outbreak of pneumonia caused by severe acute respiratory syndrome coronavirus 2 (SARS-CoV-2/ 2019-nCoV) infection was initially reported in Wuhan, Hubei Province, China. Early in 2020, the World Health Organization (WHO) announced a new name for the 2019-nCoV-caused disease: coronavirus disease 2019 (COVID-19) and declared COVID-19 to be a Public Health Emergency of International Concern (PHEIC). Cellular co-infection is a critical determinant of viral fitness and infection outcomes and plays a crucial role in shaping the host immune response to infections.

\section{Methods}

In this study, 68 public next-generation sequencing data from SARS-CoV-2 infected patients were retrieved from the NCBI Sequence Read Archive database using SRA-Toolkit. Data screening was performed using an alignment-free method based on $k$-mer mapping and extension, fastv. Taxonomic classification was performed using Kraken 2 on all reads containing one or more virus sequences other than SARS-CoV-2.

\section{Results}

SARS-CoV-2 was identified in all except three patients. Influenza type A (H7N9) virus, human immunodeficiency virus, rhabdovirus, human metapneumovirus, Human adenovirus, Human herpesvirus 1, coronavirus NL63, parvovirus, simian virus 40, and hepatitis virus genomes sequences were detected in SARS-CoV-2 infected patients. Besides, a very diverse group of bacterial populations were observed in the samples. 


\section{Non-SARS-CoV-2 genome sequences identified in}

2 clinical samples from COVID-19 infected patients:

3 Evidence for co-infections

4

5

6

7

8

13

14

15

16

172407 River Dr, Knoxville, TN 37996, USA

18

19

20

21

22

23

24

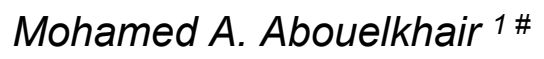

Mohamed A. Abouelkhair

Email address: mabouelk@vols.utk.edu 


\section{Abstract}

26 Background. In December 2019, an ongoing outbreak of pneumonia caused by severe acute

27 respiratory syndrome coronavirus 2 (SARS-CoV-2/ 2019-nCoV) infection was initially reported in

28 Wuhan, Hubei Province, China. Early in 2020, the World Health Organization (WHO)

29 announced a new name for the 2019-nCoV-caused disease: coronavirus disease 2019 (COVID-

30 19) and declared COVID-19 to be a Public Health Emergency of International Concern (PHEIC).

31 Cellular co-infection is a critical determinant of viral fitness and infection outcomes and plays a

32 crucial role in shaping the host immune response to infections.

33 Methods. In this study, 68 public next-generation sequencing data from SARS-CoV-2 infected

34 patients were retrieved from the NCBI Sequence Read Archive database using SRA-Toolkit.

35 Data screening was performed using an alignment-free method based on $k$-mer mapping and

36 extension, fastv. Taxonomic classification was performed using Kraken 2 on all reads containing

37 one or more virus sequences other than SARS-CoV-2.

38 Results. SARS-CoV-2 was identified in all except three patients. Influenza type A (H7N9) virus,

39 human immunodeficiency virus, rhabdovirus, human metapneumovirus, Human adenovirus,

40 Human herpesvirus 1, coronavirus NL63, parvovirus, simian virus 40, and hepatitis virus

41 genomes sequences were detected in SARS-CoV-2 infected patients. Besides, a very diverse

42 group of bacterial populations were observed in the samples.

44 Keywords: COVID-19, Co-infections, Bacteria, Influenza A virus, Human

45 immunodeficiency virus 


\section{Introduction}

50 In December 2019, the first cases of coronavirus disease 2019 (COVID-19) were possibly due

51 to a zoonotic transmission in China. It was tied to a large seafood market that also traded in live

52 wild animals (Tay et al. 2020). The causative virus, severe acute respiratory syndrome

53 coronavirus 2 (SARS-CoV-2), is capable of human-to-human transmission and rapidly spread to

54 other regions of China and other countries (World Health Organization 2020). It is now a global

55 pandemic and is a considerable concern for public health. So far, more than $16,101,367$

56 confirmed cases were diagnosed in nearly 213 countries and territories around the world and

57 two international conveyances, causing globally over 645,000 deaths (Worldometer 2020, July

58 25).

59 Coronaviruses are known to cause severe diseases in humans and animals. Of these, four human coronaviruses (229E, NL63, OC43, and HKU1) typically only infect the upper respiratory

61 tract and cause relatively minor symptoms (Fehr \& Perlman 2015). However, there are three

62 coronaviruses (severe acute respiratory syndrome coronavirus (SARS-CoV), Middle East respiratory syndrome coronavirus (MERS-CoV), and SARS-CoV-2) that can replicate in the lower respiratory tract and cause pneumonia, which can be fatal. With $79 \%$ genome sequence similarity, SARS-CoV is the closest relative to SARS-CoV-2 among human coronaviruses (Gorbalenya et al. 2020). However, of all known coronavirus sequences, SARS-CoV-2 is most

67 similar to bat coronavirus RaTG13, with a similarity of 98\% (Zhou et al. 2020).

SARS-CoV-2 pathophysiology closely parallels that of SARS-CoV infection, with active 69 inflammatory responses strongly implicated in the resulting airway damage (Wong et al. 2004).

70 Hence the extent of the disease in patients is attributed not only to the viral infection but also to

71 the host's response (Tay et al. 2020).

72 Underlying co-infections in primary infectious disease are an important variable that needs to be

73 considered but is often undetected. Remarkable developments in next-generation sequencing 
74 have recently made metagenomics, an unbiased shotgun method of analysis, a widely used tool

75 in just about every field of biology, including diagnosis of infectious diseases (Kuroda et al.

76 2012; Lecuit \& Eloit 2014). Metagenomics is powerful because it can diagnose unsuspected

77 microbial agents (Wilson et al. 2014). It directly analyzes samples in their entirety, eliminating

78 the need for prior knowledge to obtain comprehensive information. In this capacity,

79 metagenomics exceeds traditional diagnostic limitations. With the microbial genomes in hand,

80 we can now explore the possibility of using metagenomic and metatranscriptomic next-

81 generation sequencing ( $\mathrm{mNGS}$ ) directly as a screening method of other microbes in a sample

82 (Plyusnin et al. 2020).

83

84 A simple approach would be to first map sequencing reads from the sample to the reference

85 microbial genome. However, the accuracy of such an alignment-based method is relatively low

86 compared to an alignment-free approach. In the alignment-based method, genome sequences

87 from closely related viruses can lead to false-positive results (Chen et al. 2020b). Moreover, the

88 virus-specific reads obtained may not be adequate for unambiguous detection (degraded RNA,

89 incompletely target-enriched sequence library by multiple-PCR (Lundberg et al. 2013) or hybrid

90 capture (Duncavage et al. 2011), which can lead to false-negative results.

91 Fastv is an alignment-free, ultra-fast tool for detecting the microbial sequences in sequence

92 data (Chen et al. 2020b). It can identify target microorganisms using unique $k$-mers. It detects

93 SARS and other coronaviruses from sequencing data and efficiently distinguishing SARS from

94 MERS.

95

96 In this study, public next-generation sequencing data from SARS-CoV-2 infected patients were

97 analyzed by fastv using the pre-computed unique $k$-mer resources (Chen et al. 2020b).

98 Taxonomic classification was performed using Kraken 2 on all reads containing more than one 
99 microbial sequence (Wood et al. 2019). The present study's findings have confirmed the actual

100 existence of genome sequences of other microbes in SARS-CoV-2 infected patients.

101

\section{Material and Methods}

103 Computing hardware

104 Amazon Elastic Compute Cloud (EC2) instance (i.e., virtual server in the AWS cloud) was used.

105 SRA-tools package version 2.9.1 (https://github.com/ncbi/sra-tools), Kraken 2

106 (https://github.com/DerrickWood/kraken2) (Wood et al. 2019), and fastv version 0.9.0

107 (https://github.com/OpenGene/fastv) (Chen et al. 2020b) were installed within the Linux 2 EC2

108 instance.

109

\section{SRA database mining}

111 Next-generation sequencing technologies have enabled large-scale genomic surveillance of

112 SARS-CoV-2 as thousands of isolates are being sequenced worldwide and deposited in public

113 data repositories. The sequence data were downloaded as .sra files using the prefetch tool

114 (https://github.com/ncbi/sra-tools/tree/master/tools/prefetch), then extracted to. fastq files using

115 the NCBI fastq-dump tool (https://github.com/ncbi/sra-tools/tree/master/tools/fastq-dump).

116 Data sets for analysis were chosen through a keyword search of the SRA descriptions for

117 "COVID19" and downloaded between 27 January 2020 and 16 May 2020. Sequence data from

118 negative COVID-19 patients, experimental studies, and controlled access were excluded.

119 (Table S1).

120

121 Table S1: SRA sequences used in this study with the detection result for SARS-

\section{CoV-2 k-mer}

123 


\section{Sequence data pre-processing and screening using fastv}

125 Fastv, along with the pre-computed unique $k$-mer resources, was used as previously described

126 (Chen et al. 2020b). Initially, fastv performed adapter trimming, quality pruning, base correction,

127 and other pre-processing to ensure the accuracy of $k$-mer analysis on fastq input files. The fastv

128 tool identifies a target virus from sequencing data and detects any microbial sequences for

129 which a unique $k$-mer data is provided. A unique SARS-CoV-2 $k$-mer set and the SARS-CoV-2

130 reference genome were used as input files (downloaded from

131 https://github.com/OpenGene/fastv/tree/master/data) with the k-mer collections for viral and

132 microbial genomes (downloaded from http://opengene.org/microbial.kc.fasta.gz). The k-mer

133 scanning results were visualized in a figure on a single HTML page by fastv.

134

\section{Sequence data analysis using Kraken 2}

136 The results of fastv were validated with Kraken 2. Kraken 2 is the latest version of Kraken, a

137 taxonomic classification system that uses exact $k$-mer matches to achieve high accuracy and

138 rapid classification (https://github.com/DerrickWood/kraken2) (Wood et al. 2019). Kraken

139 constructs an index of all $k$-mers found in the reference genomes and assigns each $k$-mer to the

140 least common ancestor (LCA) of all species that have that $k$-mer. Then Kraken matches the $k$ -

141 mers contained in the reads to this index and eventually assigns the reads to the taxon with the

142 most fitting $k$-mers by following the path from the root of the tree (Wood et al. 2019). Through

143 lowering memory use by $85 \%$, Kraken 2 improves upon Kraken 1, enabling higher numbers of

144 genomic reference data to be used while retaining high accuracy and fivefold speed. A standard

145 database containing RefSeq complete bacterial, archaeal, and viral genomes, along with the

146 human genome and a collection of known vectors (UniVec_Core), was downloaded

147 (https://ccb.jhu.edu/software/kraken2/downloads.shtml). The database was then constructed

148 using 32 threads with the default parameters on an AWS EC2 h1.8xlarge storage optimized

149 instance with 16 dual-core hyperthreaded $2.30 \mathrm{GHz}$ CPUs and 132 gigabytes (GB) of RAM.

Peer) reviewing PDF | (2020:06:50170:2:0:NEW 13 Sep 2020) 


\section{Data visualization}

151 The results of Kraken 2 analysis were visualized with Pavian

152 (https://github.com/fbreitwieser/pavian) (Breitwieser \& Salzberg 2016) and Krona tool

153 (https://github.com/marbl/Krona/wiki) (Ondov et al. 2011), which displays hierarchical data (like

154 taxonomic assignation) in multi-layered pie charts. The Kraken 2 outputs were converted in

155 HTML format using the program ktImportTaxonomy

156 (https://github.com/marbl/Krona/tree/master/KronaTools/scripts), which parses the information

157 relative to the query ID and the taxonomy ID.

158

159 Results

160 SARS-CoV-2 Identification

161 Sequence data analysis was performed on public Illumina HiSeq/ MiSeq libraries from the NCBI

162 SRA database (Bioproject PRJNA605983) sequenced from bronchoalveolar lavage fluid from

163 five patients (WIV02, WIV04, WIV05, WIV06, and WIV07) with pneumonia at the early COVID-

16419 outbreak in Wuhan, China. Nine libraries were downloaded as .sra files using the prefetch

165 tool; then, the fastq files were extracted using the NCBI fastq-dump tool. Prior to the $k$-mer

166 analysis, sequencing adapters and low-quality bases were removed by fastv.

167 After scanning the fastq data, fastv reported the $k$-mer coverage for each microbial genome with

168 valid hits (Figure 1).

169

170 Figure 1: SARS-CoV-2 detection by fastv. SARS-CoV-2 was detected in WIV07 patient,

171 where twelve SARS-CoV-2 hits were included in the genome list and ordered by k-mer

172 coverage (99.4972\% to $99.2308 \%$ ). The number of hits (on the y-axis) of each $k$-mer key (on

173 the $\mathrm{x}$-axis) were plotted. Mismatches were highlighted in red. 
174 SARS-CoV-2 was detected in all of the 68-sequence data except SRR11772662,

175 SRR11772663, and SRR11772664 samples were negative for SARS-CoV-2. These samples

176 belong to one study (Bioproject PRJNA631042), where the research group used different

177 sequencing technologies on the same sample to find a cost-effective and highly scalable

178 method for SARS-CoV-2 sequencing. Because sequence technologies vary in reading depth

179 and coverage thresholds, fastv could not detect SARS-CoV-2 in sequenced samples with lower

180 coverage metrics.

181

182 Non-SARS-CoV-2 genome sequences were detected in COVID-19 infected patients

183 Fastv also identified influenza type A (A/Shanghai/02/2013(H7N9) and rhabdovirus genomic

184 sequences in the data from WIV02 (SRR11092058 and SRR11092063 data), WIV04

185 (SRR11092057 and SRR11092062 data), WIV05 (SRR11092061 data), WIV06 (SRR11092056

186 and SRR11092060 data), and WIV07 (SRR11092059 and SRR11092064 data) patients.

187 Influenza type A segment 4 hemagglutinin (HA) gene was detected with high coverage (100\%,

$188100 \%, 98.57 \%, 95.71 \%$, and $100 \%)$ and mean depth $(5.58,16.52,4.8,2.95$, and 881.37$)$ in

189 WIV02, WIV04, WIV05, WIV06, and WIV07 patients, respectively. Genes coding for Influenza A

190 virus polymerase, non-structural proteins, matrix proteins 1 and 2 were also identified in the

191 previously mentioned patients but with lower coverages. The genome sequence of rhabdovirus

192 was detected with coverage $(57.36,72.73,57.58,67.75$, and $53.46 \%)$ and mean depth (4.82,

$1938.13,4.84,5.08$, and 4.06) in WIV02, WIV04, WIV05, WIV06, and WIV07 patients, respectively.

194 The genome sequence of the Nipah virus was detected in WIV05, WIV06, and WIV07 patients.

195 Infection with the Nipah virus in humans causes a number of clinical manifestations that range

196 from asymptomatic (subclinical) infection to acute respiratory infection and fatal encephalitis.

197 In addition, human immunodeficiency virus, human herpesvirus 1, human T-lymphotropic virus

198 1, hepatitis virus, and simian virus 40 were found in WIV07 patient sequence data with coverage 
199 below $21 \%$. The low coverage and non-human specific pathogen hits have been overlooked

200 (Table S2).

201

202 Human coronavirus 229E genome sequence was detected (coverage $=14 \%$ and mean

203 depth=1.55) in SRR11772654 (Bioproject PRJNA631042). Human adenovirus 5 sequences

204 were detected in SRR11772660, SRR11772663, SRR11772666, SRR11772672,

205 SRR11772675 and SRR11772680 data. In addition, human adenovirus 1 sequences were

206 detected in SRR11772663 and SRR11772666 data. Parvovirus NIH-CQV genes coding for a

207 putative replication-associated protein (rep), and putative capsid protein (cap)

208 (coverage $=14.51 \%$ and mean depth=0.41) were detected in SRR10971381 (Bioproject

209 PRJNA603194) (Table S2).

210 A very diverse group of bacterial populations were observed in the COVID-19 infected patients.

211 Enterobacter hormaechei was identified in WIV02, WIV04, WIV05, WIV06, and WIV07 patients.

212 Enterobacter hormaechei is an important emerging pathogen and can cause nosocomial

213 infections, and often have resistance to multiple clinically relevant antibiotics (Monahan et al.

214 2019). Acinetobacter baumannii sequence was detected in WIV05 and WIV07 patients. $A$.

215 baumannii is one of the most successful pathogens associated with hospital-acquired infections

216 worldwide (Lee et al. 2017). Enterococcus faecalis sequence was identified in WIV04, WIV05,

217 and WIV07 patients (Table S2).

218 Coliphage phi-X174 was identified in most sequence data, which might be introduced by the

219 Illumina PhiX control library (Meyer \& Kircher 2010). Stenotrophomonas phage phiSMA7,

220 Enterobacteria phage phi80, DE3, Fels-2 and M13, Proteus virus, and Delftia phage RG-2014

221 were identified in the sequence data (Table S2).

222

\section{Kraken Taxonomic classification}


224 Taxonomic classification was performed using Kraken 2 on all reads containing more than one

225 virus sequences. The genetic data for constructing the databases were retrieved from the NCBI

226 RefSeq library. A very diverse group of viral, bacterial, and archaeal populations was observed

227 in the samples. A taxonomic classification that was obtained from WIV04, WIV06, and WIV07

228 patients revealed a dominance of Bacteria (7\%, 5\%, and $59 \%$, respectively), followed by

229 Viruses $(0.3 \%, 0.3 \%$, and $0.3 \%$, respectively) while Archaea was lower than $0.02 \%$ in all the

230 patients. Among viral communities, influenza type A (8\%), rather than SARS-CoV-2 (2\%), was

231 found to be dominant in the WIV07 patient, which is consistent with fastv result (Figure 2).

232 SARS-CoV-2, rhabdovirus, and influenza type A dominated the sequence data from WIV06

233 patient (Figure 3) and WIV04 patient (Figure 4).

234 A rank code, indicating unclassified $(U)$, domain $(D)$, kingdom $(K)$, phylum $(P)$, class $(C)$, order

$235(\mathrm{O})$, family $(\mathrm{F})$, genus $(\mathrm{G})$, or species $(\mathrm{S})$ was used.

236

237 Figure 2: The Krona pie chart of the viral taxonomy of the WIV07 patient. Viruses identified

238 from Kraken 2 analysis of samples collected from SRR11092059 patients were visualized using

239 the krona tool. Among viral communities, influenza type A (8\%), SARS-CoV-2 (2\%), and

240 rhabdovirus $(0.6 \%)$ dominated the sequence data from WIV07 patient.

242 Figure 3: The Krona pie chart of the viral taxonomy of the WIV06 patient. Viruses identified

243 from Kraken 2 analysis of samples collected from SRR11092060 patients were visualized using

244 the krona tool. Among viral communities, SARS-CoV-2 (1\%), rhabdovirus (0.6\%), and influenza

245 type A (0.4\%) dominated the sequence data from WIV06 patient.

247 Figure 4: The Krona pie chart of the viral taxonomy of the WIV04 patient. Viruses identified

248 from Kraken 2 analysis of samples collected from SRR11092062 patients were visualized using 
249 the krona tool. Among viral communities, SARS-CoV-2 (1\%), rhabdovirus $(0.8 \%)$, and influenza

250 type $\mathrm{A}(0.4 \%)$ dominated the sequence data from WIV04 patient.

252 Acinetobacter baumannii and Enterobacter species genomic sequences were detected in

253 SRR11092059 (Figure 5), SRR11092060 (Figure 6), SRR11092061, SRR11092062 (Figure

254 7), and SRR11092063 sequence data. By using Kraken2, a tool for read taxonomy, many

255 bacterial species were identified that most of them present in the normal flora and rarely cause

256 problems. However, compared to the fastv result, the Pasteurella multocida sequence was

257 detected in SRR11092062 (Figure 7) and SRR11092063 sequence date. The genome

258 sequence of Staphylococcus aureus was detected in SRR11092059 data (Figure 5).

259

260

Figure5: Bacteria Identified in SRR11092059. Sankey diagrams of Kraken 2 report results obtained from SRR11092059. The width of the flow is proportional to the number of reads. The number above each node is the number of $k$-mer hits. A rank code, indicating domain (D), phylum $(P)$, family $(F)$, genus $(G)$, or species $(S)$ was used.

264

Figure 6: Bacteria Identified in SRR11092060. Sankey diagrams of Kraken 2 report results obtained from SRR11092060. The width of the flow is proportional to the number of reads. The number above each node is the number of $k$-mer hits. A rank code, indicating domain (D), phylum $(P)$, family $(F)$, genus $(G)$, or species $(S)$ was used.

Figure 7: Bacteria Identified in SRR11092062. Sankey diagrams of Kraken 2 report results

271 obtained from SRR11092062. The width of the flow is proportional to the number of reads. The

272 number above each node is the number of $k$-mer hits. A rank code, indicating domain (D),

273 phylum $(P)$, family $(F)$, genus $(G)$, or species $(S)$ was used. 
275 The most abundant bacterial phylum was Bacteroidetes, followed by Firmicutes phylum in

276 SRR10971381 sequence data (Figure 8).

277 Figure 8: Sankey diagrams of Kraken 2 report results obtained from SRR10971381. The

278 width of the flow is proportional to the number of reads. The number above each node is the

279 number of $k$-mer hits. A rank code, indicating domain (D), kingdom (K), phylum (P), family $(F)$,

280 genus (G), or species (S) was used.

281

\section{Discussion}

283 The importance of detecting co-infections is becoming more recognized (Griffiths et al. 2011; Li

284 \& Zhou 2013), but it remains challenging to get such information. The source of clinical samples

285 and the sequencing technology can be inferior in co-infection detection (Birdsell et al. 2018b).

286 A better understanding of co-infection's prevalence is required, partly because co-infecting

287 pathogens can interact with each other directly or indirectly via the host's resources or immune

288

system (Cox 2001; Griffiths et al. 2011). These interactions within co-infected hosts can alter the

289

transmission, clinical progression, and control of multiple infectious diseases as compared to

290

single pathogen species infection (Chiodini 2001; Griffiths et al. 2011; Palacios et al. 2009).

291 Recent studies indicate that co-infection's adverse effects are more common than those with

292 no-effects or positive impacts on human health (Pullan \& Brooker 2008).

293 The underdiagnosis of co-infections is attributed to a lack of clinical suspicion, common

294 symptoms, and the fact that conventional methods have little capacity to detect co-infections in

295 the absence of a priori knowledge.

296

297 Co-infection can potentially affect the performance of laboratory testing for coronavirus disease

298 2019. A previous study (Lai et al. 2020) showed that reverse transcription polymerase chain

299 reaction (RT -PCR) could not detect SARS-CoV-2 in two patients co-infected with influenza A 
300 virus. Some researchers have suggested that inadequate viral specimens, the use of improperly

301 validated assay, timing and methods of collecting specimens, the presence of mutations at the

302 primer binding site, and co-infection with other viruses might be responsible (Arevalo-Rodriguez

303 et al. 2020; Kucirka et al. 2020; Lai et al. 2020; Li et al. 2020). Based on a limited number of

304 observational studies, it was found that the false-negative rate for SARS-CoV-2 RT-PCR was

305 20\% (Lai et al. 2020; Li et al. 2020).

306

307 Given these considerations, COVID-19 might be underdiagnosed, especially during the

308 influenza season, since typical clinical symptoms of COVID-19, including fever, cough, and

309 dyspnea, resemble those of influenza (Chen et al. 2020a; Wu et al. 2020).

310 Therefore, exploring new diagnostic approaches is essential to advance understanding of co-

311 infection contribution to disease manifestations and treatment responses (Birdsell et al. 2018a).

312 Remarkable developments in next-generation sequencing have recently made metagenomics,

313 an unbiased shotgun method of analysis, a widely used tool in just about every field of biology,

314 including diagnosis of infectious diseases (Kuroda et al. 2012; Lecuit \& Eloit 2014).

315

316 In this study, using $k$-mer based tools (fastv and Kraken 2), genome sequences of various

317 microorganisms, including the SARS-CoV-2 virus, were detected. The accuracy of such an

318 alignment-free method is relatively high compared to an alignment-based approach. Fastv is an

319 alignment-free, ultra-fast tool for detecting the microbial sequences in sequence data using

320 unique $k$-mers (Chen et al. 2020b). To validate the results obtained from fastv analysis, Kraken

3212 was used. Kraken 2 is the latest version of Kraken, a taxonomic classification system that

322 uses exact $k$-mer matches to achieve high accuracy and rapid classification (Wood et al. 2019).

323 Kraken 2 is fast and requires less memory than Kraken 1. However, the standard database

324 construction requires approximately 100 gigabytes (GB) of disk space and random-access

325 memory (RAM) of more than 30 GB. For that purpose, an AWS EC2 h1.8xlarge storage

Peer) reviewing PDF | (2020:06:50170:2:0:NEW 13 Sep 2020) 
326 optimized instance with 16 dual-core hyperthreaded $2.30 \mathrm{GHz}$ CPUs and $132 \mathrm{~GB}$ of RAM was

327 used.

328 Multidrug-resistant bacteria associated with hospital-acquired infections worldwide, such as $E$.

329 hormaechei, S. aureus, P. multocida, and A. baumannii, were detected in COVID-19 infected

330 patients (Lee et al. 2017; Monahan et al. 2019). Most of the infections caused by the previously

331 mentioned bacteria occur in critically ill and or immunocompromised patients in the intensive

332 care unit (ICU) setting (Fournier et al. 2006).

333

334 Understanding the nature and consequences of co-infection is essential for accurate estimates

335 of infectious disease burden. In particular, more systematic data on infectious diseases would

336 also help measure the extent of co-infection on human health. Increased knowledge of the risk

337 factors, the conditions in which co-infecting pathogens interact, and the mechanisms behind

338 these interactions, particularly in experimental studies, will also help develop and evaluate

339 infectious disease management programs.

340

\section{Conclusions}

342 In this study, screening of 68 public next-generation sequencing data from SARS-CoV-2

343 infected patients was performed using fastv and Kraken 2. Multiple viruses, including the SARS-

344 CoV-2 virus, genome sequences were detected. Further large-sample studies are warranted to

345 investigate the prevalence of COVID-19 co-infection, the impact of co-infection on the host

346 immune system of COVID-19 patients, and their role in disease progression.

347

References

349

Arevalo-Rodriguez I, Buitrago-Garcia D, Simancas-Racines D, Zambrano-Achig $P$, del Campo R, Ciapponi A, Sued O, Martinez-Garcia L, Rutjes A, Low N, Bossuyt PM, Perez-Molina JA, and Zamora J. 2020. FALSE-NEGATIVE RESULTS OF INITIAL RT-PCR ASSAYS FOR COVID- 
352

353

354

355

356

357

358

359

360

361

362

363

364

365

366

367

368

369

370

371

372

373

374

375

376

377

378

379

380

381

382

383

384

385

386

387

388

389

390

391

392

393

394

19: A SYSTEMATIC REVIEW. medRxiv:2020.2004.2016.20066787. 10.1101/2020.04.16.20066787

Birdsell DN, Özsürekci Y, Rawat A, Aycan AE, Mitchell CL, Sahl JW, Johansson A, Colman RE, Schupp JM, Ceyhan M, Keim PS, and Wagner DM. 2018a. Coinfections identified from metagenomic analysis of cervical lymph nodes from tularemia patients. BMC infectious diseases 18:319-319. 10.1186/s12879-018-3218-2

Birdsell DN, Özsürekci Y, Rawat A, Aycan AE, Mitchell CL, Sahl JW, Johansson A, Colman RE, Schupp JM, Ceyhan M, Keim PS, and Wagner DM. 2018b. Coinfections identified from metagenomic analysis of cervical lymph nodes from tularemia patients. BMC infectious diseases 18:319. 10.1186/s12879-018-3218-2

Breitwieser FP, and Salzberg SL. 2016. Pavian: Interactive analysis of metagenomics data for microbiomics and pathogen identification. bioRxiv:084715. 10.1101/084715

Chen N, Zhou M, Dong X, Qu J, Gong F, Han Y, Qiu Y, Wang J, Liu Y, Wei Y, Xia J, Yu T, Zhang X, and Zhang L. 2020a. Epidemiological and clinical characteristics of 99 cases of 2019 novel coronavirus pneumonia in Wuhan, China: a descriptive study. Lancet 395:507-513. 10.1016/S0140-6736(20)30211-7

Chen S, He C, Li Y, Li Z, and Melançon CE. 2020b. A Computational Toolset for Rapid Identification of SARS-CoV-2, other Viruses, and Microorganisms from Sequencing Data. bioRxiv:2020.2005.2012.092163. 10.1101/2020.05.12.092163

Chiodini PL. 2001. Chemotherapy for patients with multiple parasitic infections. Parasitology 122:S83-589.

Cox FE. 2001. Concomitant infections, parasites and immune responses. Parasitology 122 Suppl:S23-38. 10.1017/s003118200001698x

Duncavage EJ, Magrini V, Becker N, Armstrong JR, Demeter RT, Wylie T, Abel HJ, and Pfeifer JD. 2011. Hybrid capture and next-generation sequencing identify viral integration sites from formalin-fixed, paraffin-embedded tissue. J Mol Diagn 13:325-333. 10.1016/j.jmoldx.2011.01.006

Fehr AR, and Perlman S. 2015. Coronaviruses: an overview of their replication and pathogenesis. Coronaviruses: Springer, 1-23.

Fournier PE, Richet $\mathrm{H}$, and Weinstein RA. 2006. The epidemiology and control of Acinetobacter baumannii in health care facilities. Clinical infectious diseases 42:692-699.

Gorbalenya AE, Baker SC, Baric RS, de Groot RJ, Drosten C, Gulyaeva AA, Haagmans BL, Lauber C, Leontovich AM, Neuman BW, Penzar D, Perlman S, Poon LLM, Samborskiy DV, Sidorov IA, Sola I, Ziebuhr J, and Coronaviridae Study Group of the International Committee on Taxonomy of V. 2020. The species Severe acute respiratory syndrome-related coronavirus: classifying 2019-nCoV and naming it SARS-CoV-2. Nature Microbiology 5:536-544. 10.1038/s41564-020-0695-z

Griffiths EC, Pedersen AB, Fenton A, and Petchey OL. 2011. The nature and consequences of coinfection in humans. Journal of Infection 63:200-206. https://doi.org/10.1016/i.jinf.2011.06.005

Kucirka LM, Lauer SA, Laeyendecker O, Boon D, and Lessler J. 2020. Variation in False-Negative Rate of Reverse Transcriptase Polymerase Chain Reaction-Based SARS-CoV-2 Tests by Time Since Exposure. Annals of Internal Medicine 173:262-267. 10.7326/M20-1495

Peer) reviewing PDF | (2020:06:50170:2:0:NEW 13 Sep 2020) 
395

396

397

398

399

400

401

402

403

404

405

406

407

408

409

410

411

412

413

414

415

416

417

418

419

420

421

422

423

424

425

426

427

428

429

430

431

432

433

434

435

436

437

Kuroda M, Sekizuka T, Shinya F, Takeuchi F, Kanno T, Sata T, and Asano S. 2012. Detection of a possible bioterrorism agent, Francisella sp., in a clinical specimen by use of nextgeneration direct DNA sequencing. J Clin Microbiol 50:1810-1812. 10.1128/JCM.0671511

Lai C-C, Wang C-Y, and Hsueh P-R. 2020. Co-infections among patients with COVID-19: The need for combination therapy with non-anti-SARS-CoV-2 agents? Journal of Microbiology, Immunology and Infection. https://doi.org/10.1016/j.jmii.2020.05.013

Lecuit M, and Eloit M. 2014. The diagnosis of infectious diseases by whole genome next generation sequencing: a new era is opening. Front Cell Infect Microbiol 4:25. 10.3389/fcimb.2014.00025

Lee C-R, Lee JH, Park M, Park KS, Bae IK, Kim YB, Cha C-J, Jeong BC, and Lee SH. 2017. Biology of Acinetobacter baumannii: Pathogenesis, Antibiotic Resistance Mechanisms, and Prospective Treatment Options. Frontiers in Cellular and Infection Microbiology 7:55.

Li D, Wang D, Dong J, Wang N, Huang H, Xu H, and Xia C. 2020. False-Negative Results of RealTime Reverse-Transcriptase Polymerase Chain Reaction for Severe Acute Respiratory Syndrome Coronavirus 2: Role of Deep-Learning-Based CT Diagnosis and Insights from Two Cases. Korean J Radiol 21:505-508.

Li XX, and Zhou XN. 2013. Co-infection of tuberculosis and parasitic diseases in humans: a systematic review. Parasit Vectors 6:79. 10.1186/1756-3305-6-79

Lundberg DS, Yourstone S, Mieczkowski P, Jones CD, and Dangl JL. 2013. Practical innovations for high-throughput amplicon sequencing. Nat Methods 10:999-1002. 10.1038/nmeth.2634

Meyer M, and Kircher M. 2010. Illumina Sequencing Library Preparation for Highly Multiplexed Target Capture and Sequencing. Cold Spring Harbor Protocols 2010:pdb.prot5448.

Monahan LG, DeMaere MZ, Cummins ML, Djordjevic SP, Roy Chowdhury P, and Darling AE. 2019. High contiguity genome sequence of a multidrug-resistant hospital isolate of Enterobacter hormaechei. Gut Pathogens 11:3. 10.1186/s13099-019-0288-7

Ondov BD, Bergman NH, and Phillippy AM. 2011. Interactive metagenomic visualization in a Web browser. BMC Bioinformatics 12:385. 10.1186/1471-2105-12-385

Palacios G, Hornig M, Cisterna D, Savji N, Bussetti AV, Kapoor V, Hui J, Tokarz R, Briese T, Baumeister E, and Lipkin WI. 2009. Streptococcus pneumoniae coinfection is correlated with the severity of H1N1 pandemic influenza. PLoS One 4:e8540. 10.1371/journal.pone.0008540

Plyusnin I, Kant R, Jääskeläinen AJ, Sironen T, Holm L, Vapalahti O, and Smura T. 2020. Novel NGS Pipeline for Virus Discovery from a Wide Spectrum of Hosts and Sample Types. bioRxiv:2020.2005.2007.082107. 10.1101/2020.05.07.082107

Pullan R, and Brooker S. 2008. The health impact of polyparasitism in humans: are we underestimating the burden of parasitic diseases? Parasitology 135:783-794.

Tay MZ, Poh CM, Renia L, MacAry PA, and Ng LFP. 2020. The trinity of COVID-19: immunity, inflammation and intervention. Nat Rev Immunol. 10.1038/s41577-020-0311-8

Wilson MR, Naccache SN, Samayoa E, Biagtan M, Bashir H, Yu G, Salamat SM, Somasekar S, Federman S, and Miller S. 2014. Actionable diagnosis of neuroleptospirosis by nextgeneration sequencing. New England Journal of Medicine 370:2408-2417.

Peer] reviewing PDF | (2020:06:50170:2:0:NEW 13 Sep 2020) 
438 Wong CK, Lam CW, Wu AK, Ip WK, Lee NL, Chan IH, Lit LC, Hui DS, Chan MH, Chung SS, and Sung JJ. 2004. Plasma inflammatory cytokines and chemokines in severe acute respiratory syndrome. Clin Exp Immunol 136:95-103. 10.1111/j.1365-2249.2004.02415.x

Wood DE, Lu J, and Langmead B. 2019. Improved metagenomic analysis with Kraken 2. Genome Biology 20:257. 10.1186/s13059-019-1891-0

World Health Organization. 2020. WHO Director-General's statement on IHR Emergency Committee on Novel Coronavirus (2019-nCoV).

Worldometer. 2020, July 25. Covid-19 coronavirus pandemic.

Wu X, Cai Y, Huang X, Yu X, Zhao L, Wang F, Li Q, Gu S, Xu T, Li Y, Lu B, and Zhan Q. 2020. Coinfection with SARS-CoV-2 and Influenza A Virus in Patient with Pneumonia, China. Emerg Infect Dis 26:1324-1326. 10.3201/eid2606.200299

Zhou P, Yang XL, Wang XG, Hu B, Zhang L, Zhang W, Si HR, Zhu Y, Li B, Huang CL, Chen HD, Chen J, Luo Y, Guo H, Jiang RD, Liu MQ, Chen Y, Shen XR, Wang X, Zheng XS, Zhao K, Chen QJ, Deng F, Liu LL, Yan B, Zhan FX, Wang YY, Xiao GF, and Shi ZL. 2020. A pneumonia 10.1038/s41586-020-2012-7 


\section{Figure 1}

SARS-CoV-2 detection by fastv.

SARS-CoV-2 was detected in WIV07 patient, where twelve SARS-CoV-2 hits were included in the genome list and ordered by $k$-mer coverage (99.4972\% to $99.2308 \%)$. Mismatches were highlighted in red.

Unique k-mer hits (724 k-mer keys)

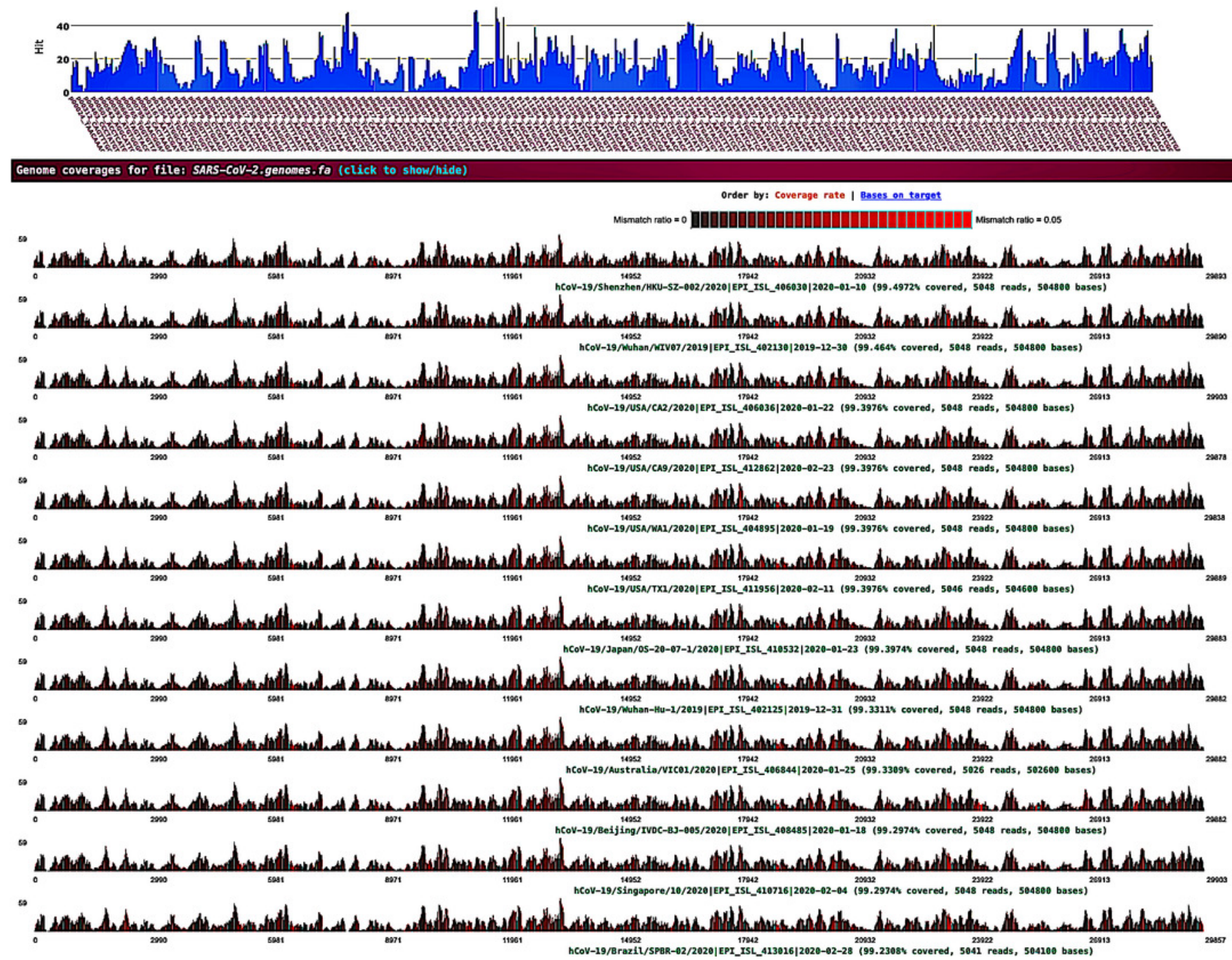


Figure 2

The Krona pie chart of the viral taxonomy of the WIV07 patient.

Viruses identified from kraken 2 analysis of samples collected from SRR11092059 patients were visualized using krona tool. Among viral communities, influenza type A (8\%), SARS-

CoV-2 $(2 \%)$ and rhabdovirus (0.6\%) dominated the sequence data from WIV07 patient.

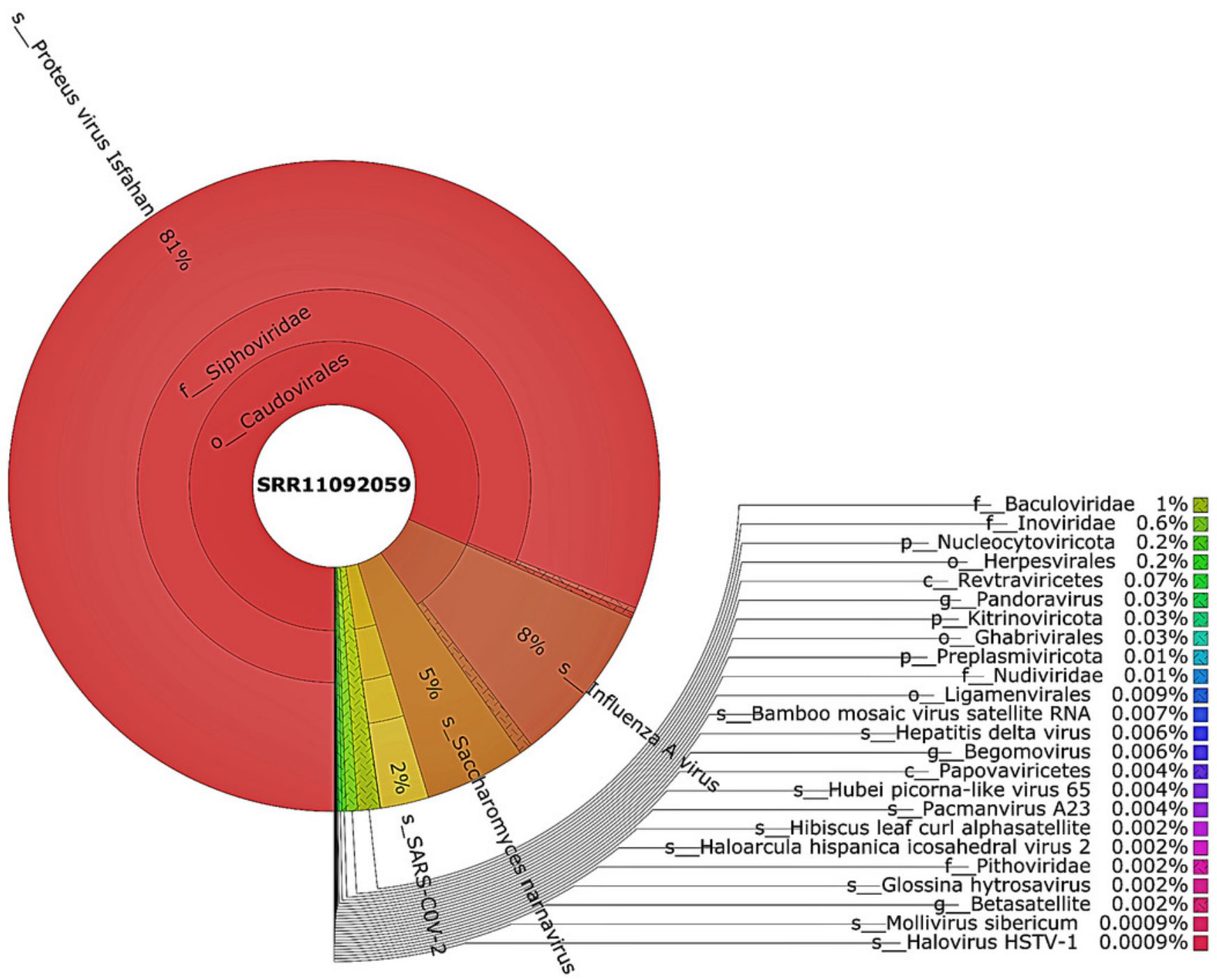




\section{Figure 3}

The Krona pie chart of the viral taxonomy of the WIV06 patient.

Viruses identified from kraken 2 analysis of samples collected from SRR11092060 patients were visualized using krona tool. Among viral communities, SARS-CoV-2 (1\%), rhabdovirus (0.6\%) and influenza type A (0.4\%) dominated the sequence data from WIV06 patient.

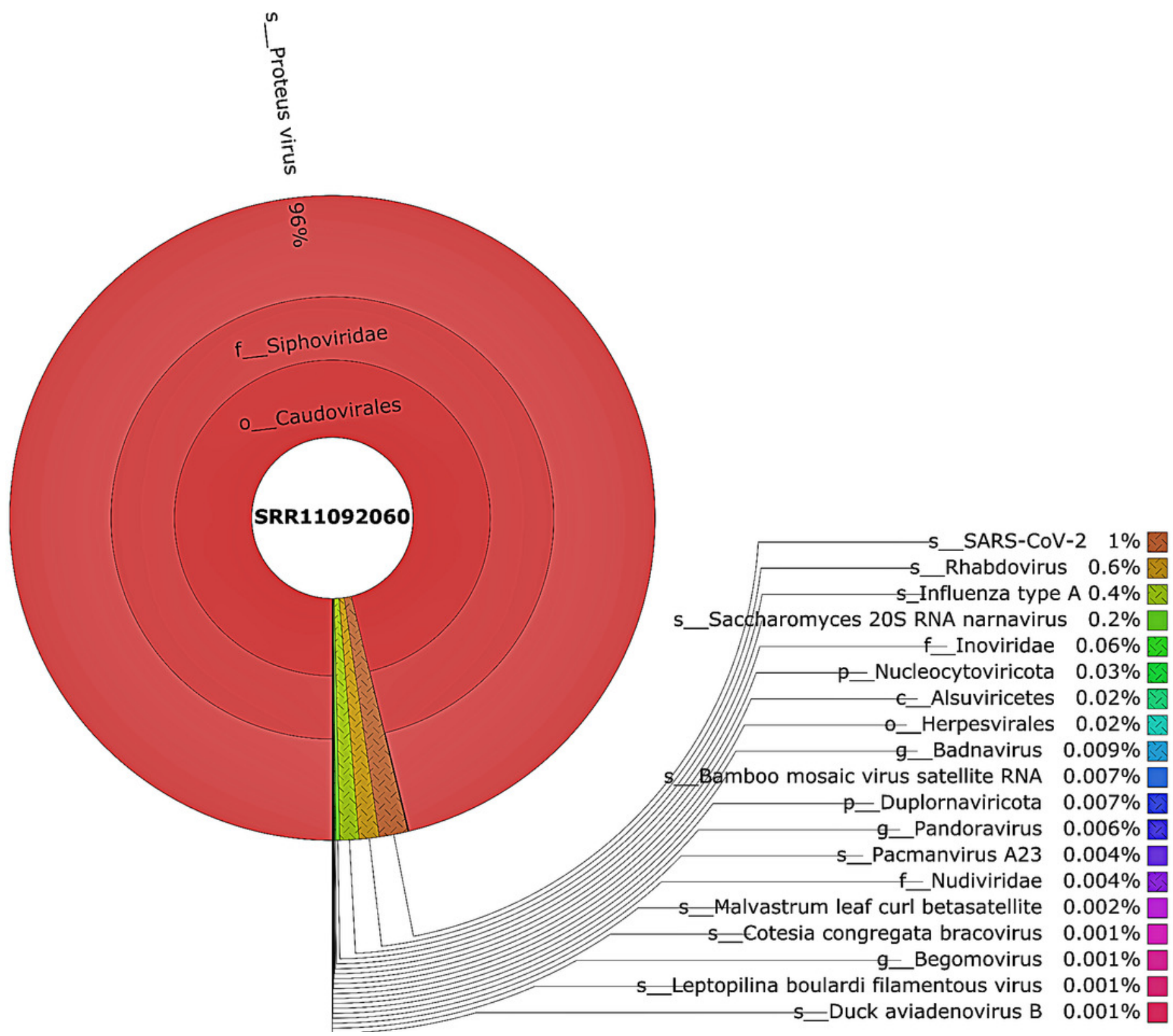




\section{Figure 4}

The Krona pie chart of the viral taxonomy of the WIV04 patient.

Viruses identified from kraken 2 analysis of samples collected from SRR11092062 patients were visualized using krona tool. Among viral communities, SARS-CoV-2 (1\%), rhabdovirus $(0.8 \%)$ and influenza type A $(0.4 \%)$ dominated the sequence data from WIV04 patient.

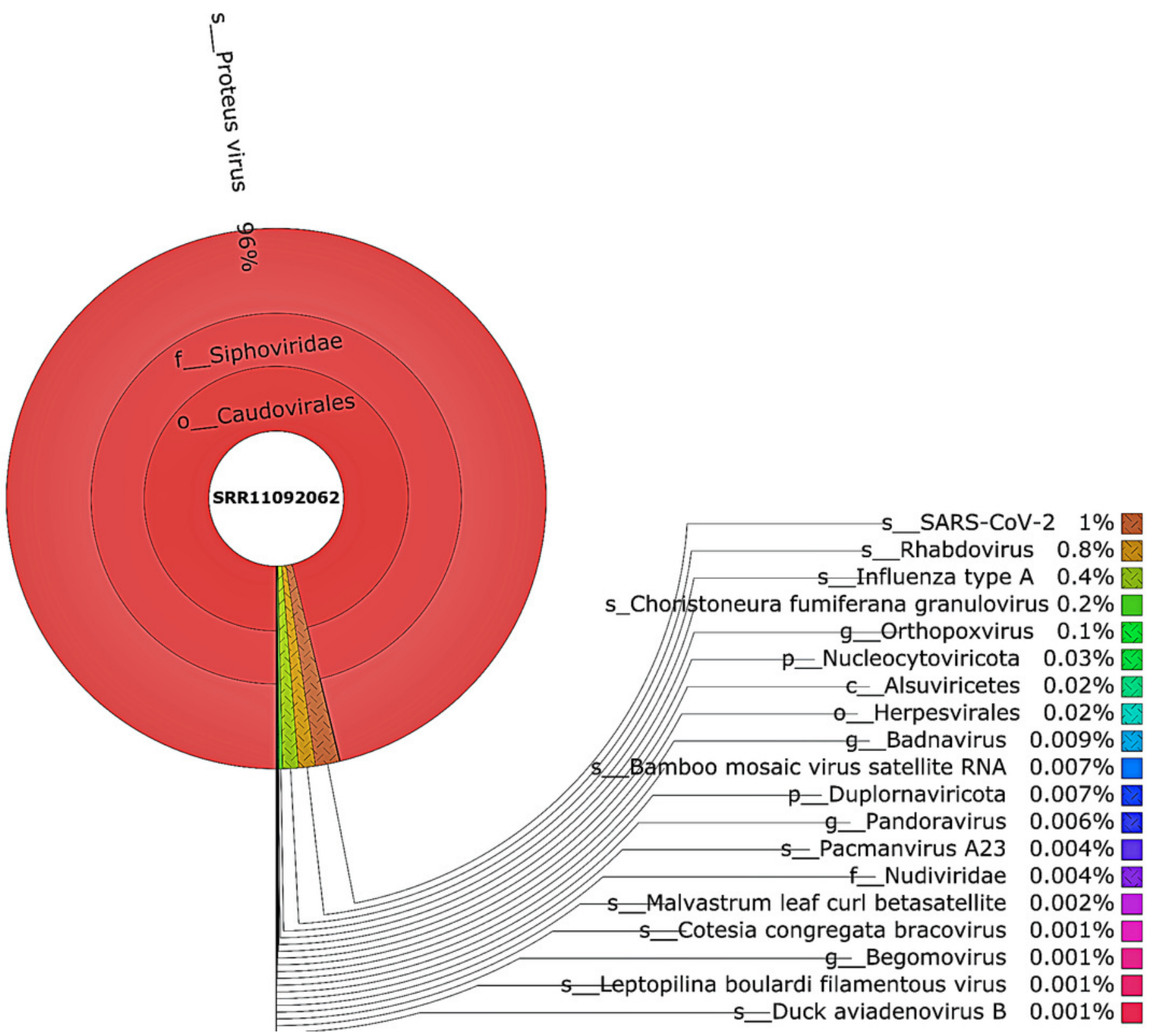




\section{Figure 5}

Bacteria Identified in SRR11092059

Sankey diagrams of Kraken 2 report results obtained from SRR11092059. The width of the flow is proportional to the number of reads. The number above each node is the number of $k$ mer hits. A rank code, indicating domain (D), phylum (P), family $(F)$, genus $(G)$, or species $(S)$ was used.

\section{SRR11092059}

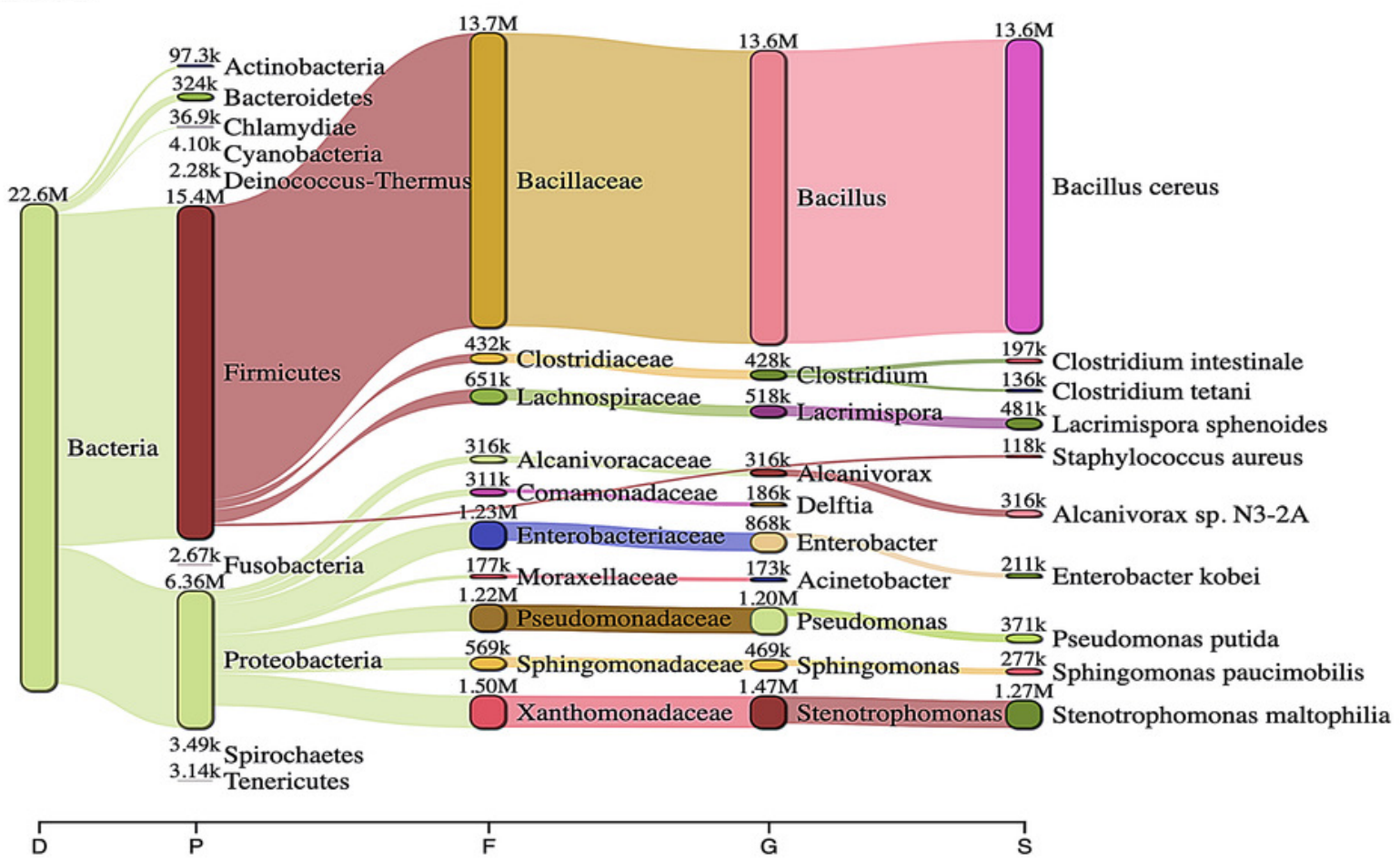




\section{Figure 6}

\section{Bacteria Identified in SRR11092060}

Sankey diagrams of Kraken 2 report results obtained from SRR11092060. The width of the flow is proportional to the number of reads. The number above each node is the number of $k$ mer hits. A rank code, indicating domain (D), phylum (P), family $(F)$, genus $(G)$, or species $(S)$ was used.

\section{SRR11092060}

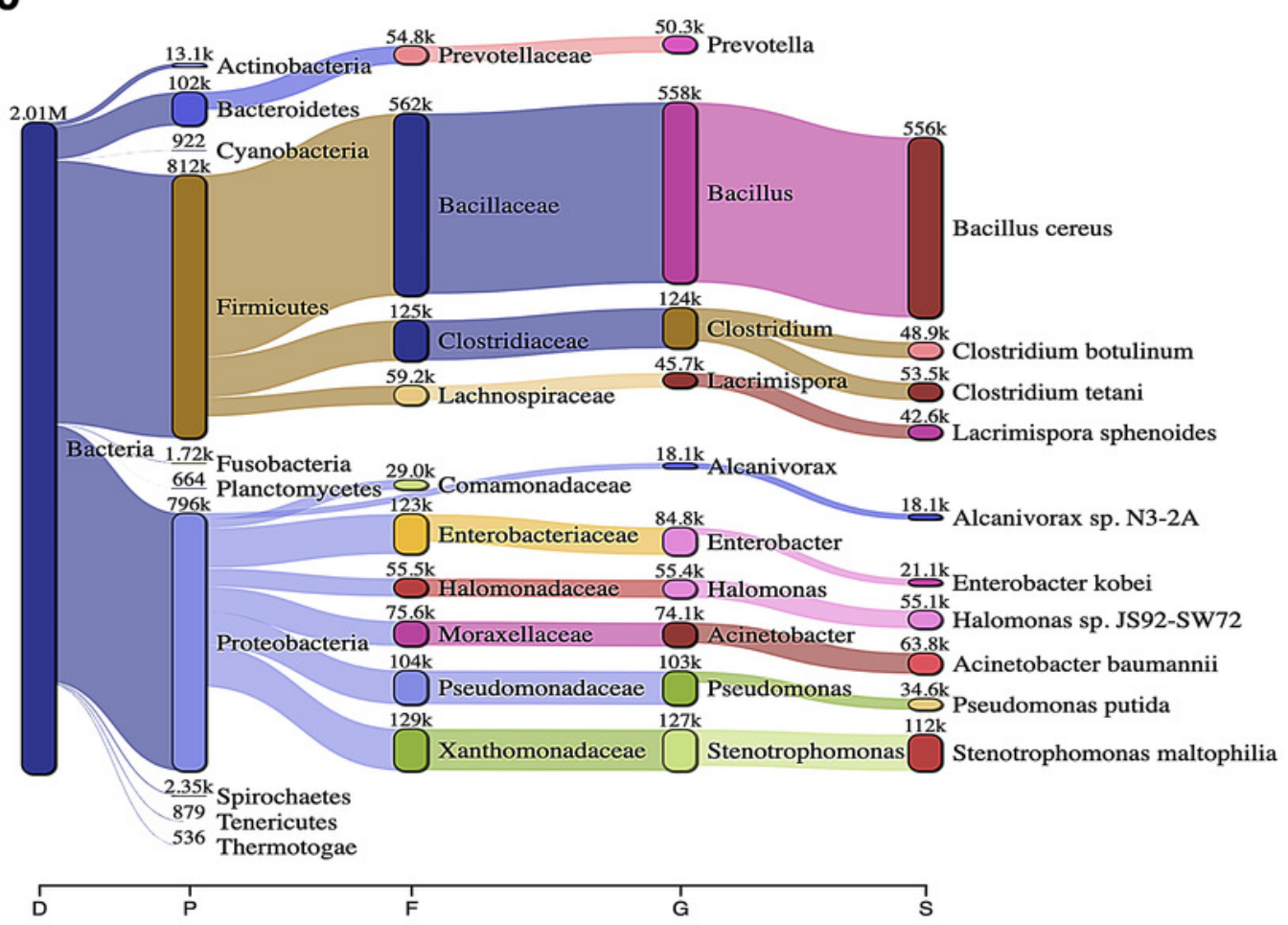


Figure 7

Bacteria Identified in SRR11092062

Sankey diagrams of Kraken 2 report results obtained from SRR11092062. The width of the flow is proportional to the number of reads. The number above each node is the number of $k$ mer hits. A rank code, indicating domain (D), phylum (P), family $(F)$, genus $(G)$, or species $(S)$ was used.

SRR11092062

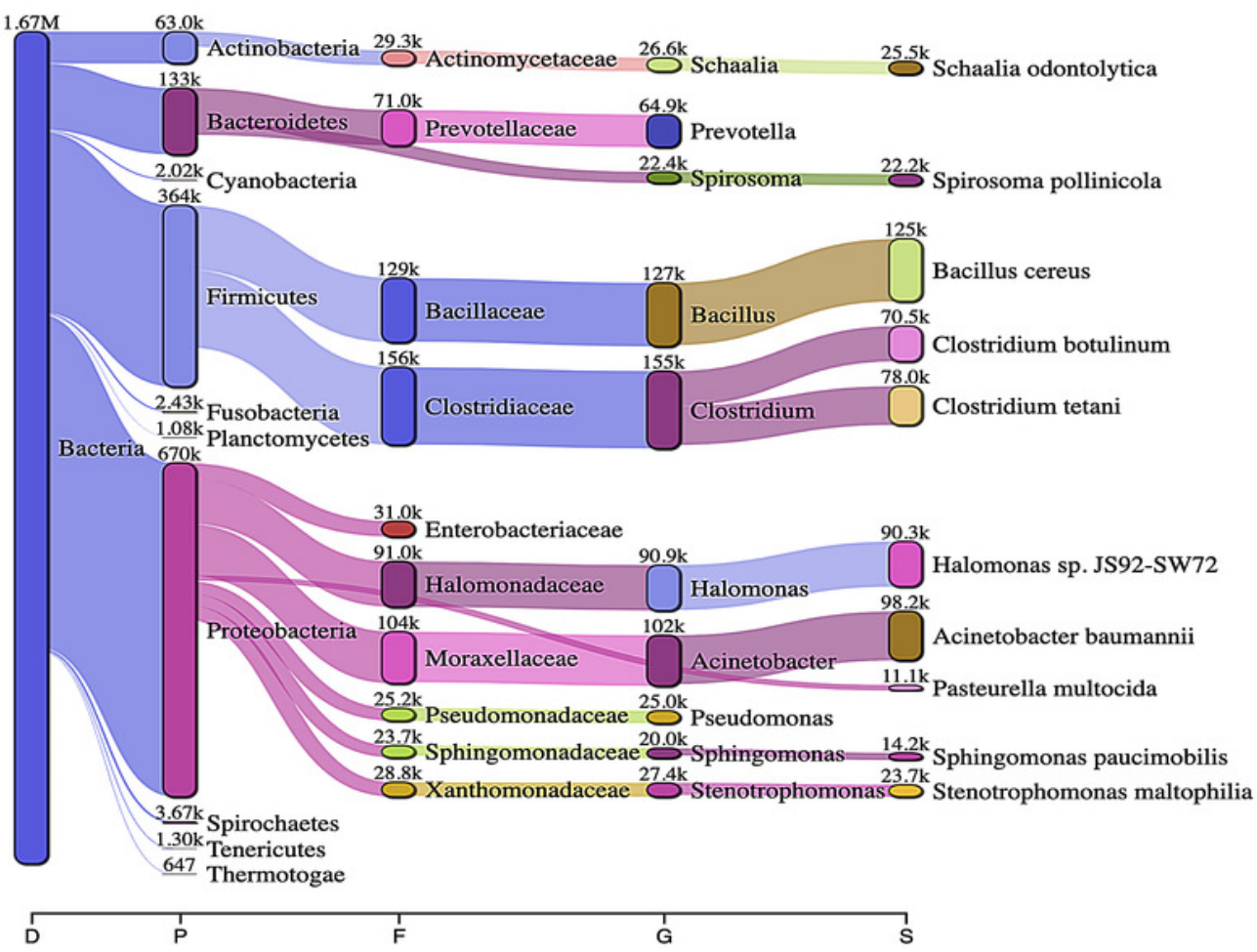


Figure 8

Sankey diagrams of Kraken 2 report results obtained from SRR10971381

The width of the flow is proportional to the number of reads. The number above each node is the number of $k$-mer hits. A rank code, indicating domain (D), kingdom (K), phylum (P), family $(F)$, genus $(G)$, or species (S) was used.

\section{SRR10971381}

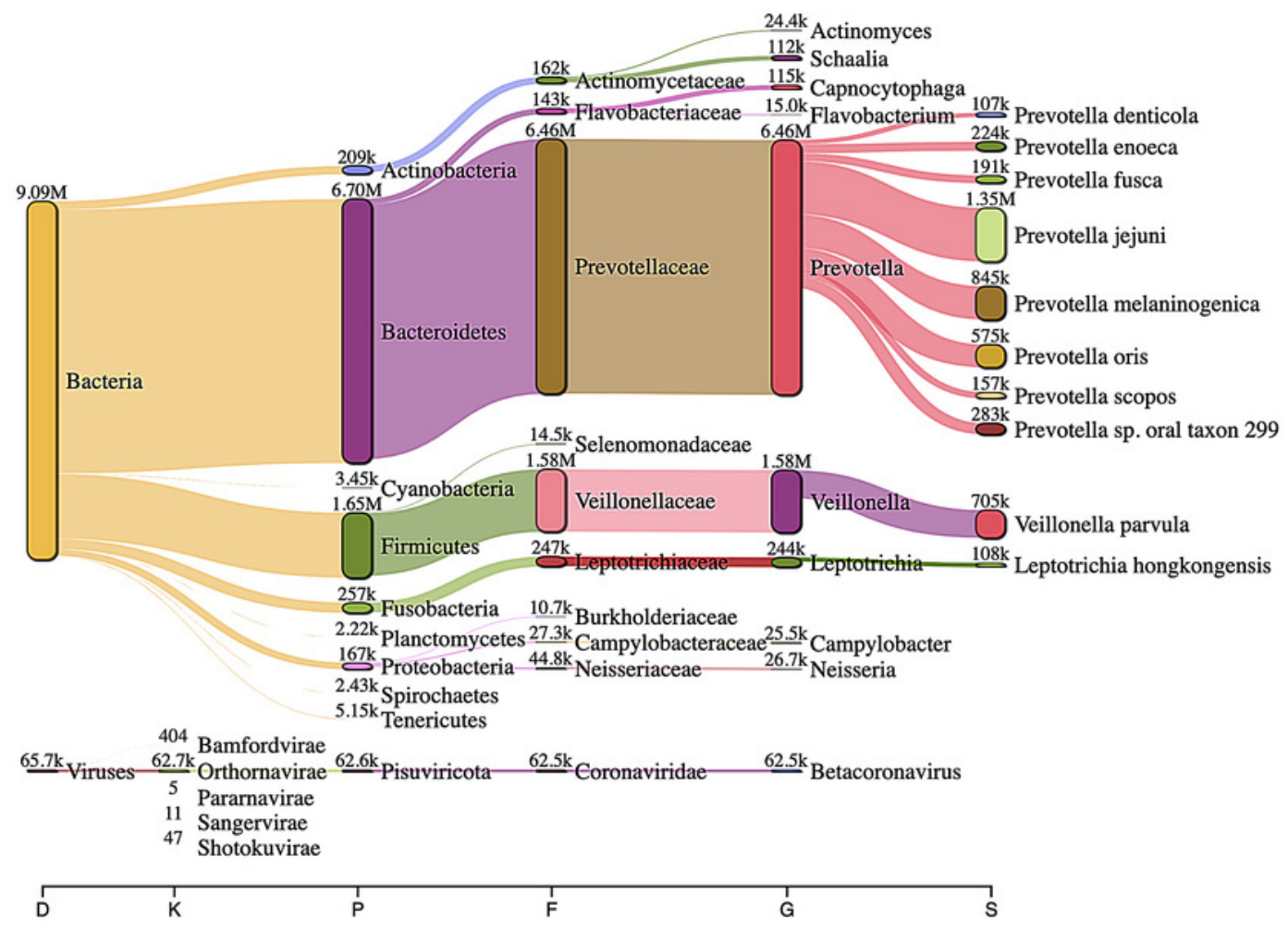

